\title{
Integrated analyses of a major histocompatibility complex, methylation and transcribed ultra-conserved regions in systemic lupus erythematosus
}

\author{
HUA LIN $^{1 *}$, WEIGUO SUI ${ }^{1 *}$, QIUPEI TAN ${ }^{1}$, JIEJING CHEN $^{1}$, YUE ZHANG ${ }^{1}$, MINGLIN OU $^{1}$, \\ WEN XUE ${ }^{1}$, FENGYAN LI ${ }^{1}$, CUIHUI CAO ${ }^{1}$, YUFENG SUN ${ }^{1}$ and YONG DAI ${ }^{2}$ \\ ${ }^{1}$ Nephrology Department of 181st Hospital, Guangxi Key Laboratory of Metabolic Diseases Research, \\ Guilin, Guangxi 541002; ${ }^{2}$ The Second Clinical Medical College of Jinan University \\ (Shenzhen People's Hospital), Shenzhen, Guangdong 518020, P.R. China
}

Received May 2, 2015; Accepted October 5, 2015

DOI: $10.3892 /$ ijmm.2015.2416

\begin{abstract}
Systemic lupus erythematosus (SLE) is a multifactorial autoimmune disease which affects different organs and systems that, has a complex genetic inheritance, and is affected by both epigenetic and environmental risk factors. Previous studies on SLE have lacked the statistical power and genetic resolution to fully determine the influence of major histocompatibility complex (MHC) on SLE. In this study, in order to determine this influence, a total of 15 patients with SLE and 15 healthy controls were enrolled. MHC region capture technology, hMeDIP-chip, transcribed ultra-conserved region (T-UCR) microarray and bioinformatics analysis were utilized for both groups. The results revealed methylated $\mathrm{CpG}$ enrichment at 6 loci in the MHC segment of SLE. We found 4 single-nucleotide polymorphisms (SNPs) in the CpG promoter of human leukocyte antigen-B (HLA-B) and 2 SNPs in chr6:29521110-29521833. No significant GO term or KEGG pathway enrichment was noted for an immunecorrelated process in the SLE patients for the corresponding CpG-methylated genes. In this study, T-UCR was not discovered in the MHC segment. The analysis of SNPs (rs1050683, rs12697943,rs17881210,rs1065378,rs17184255 and rs16895070) and gene expression in peripheral blood lymphocytes indicated that these SNPs were associated with the occurrence of SLE. Further studies are warranted to examine the roles of these SNPs in the pathogenesis of SLE. Integrative analysis technology provided a view of the molecular signaling pathways in SLE.
\end{abstract}

Correspondence to: Professor Yong Dai, The Second Clinical Medical College of Jinan University (Shenzhen People's Hospital), Shenzhen, Guangdong 518020, P.R. China

E-mail: daiyong2222@gmail.com; daiyong22@aliyun.com

${ }^{*}$ Contributed equally

Key words: major histocompatibility complex, methylation, transcribed ultra-conserved regions, systemic lupus erythematosus, bioinformatics

\section{Introduction}

Systemic lupus erythematosus (SLE) is a systemic autoimmune disease that affects different organs and systems and has a complex genetic inheritance (1). SLE has a complex etiology and is affected by both genetic and environmental factors (2). The major histocompatibility complex (MHC) located on chromosome $6 \mathrm{p} 21$ is one of the key factors that contribute to the development of SLE (3). The human leukocyte antigen (HLA) has been shown to be associated with susceptibility to SLE. Genome-wide association studies have demonstrated that variants within the MHC region confer the greatest genetic risk of developing SLE in European and Chinese populations. However, the causal variants remain elusive due to the tight linkage disequilibrium across disease-associated MHC haplotypes, the highly polymorphic nature of several MHC genes, and the heterogeneity of SLE phenotypes. The loci include HLA-DPB1, HLA-G and MSH5, which are independent of each other and $H L A-D R B 1$ alleles. These data highlight the usefulness of mapping disease susceptibility loci using a transancestral approach, particularly in a region as complex as the $\mathrm{MHC}$, and offer a springboard for further fine-mapping, resequencing and transcriptomic analysis (4). Certain studies have suggested an association between MHC class I and II (HLA- $A^{*} 29, H L A-B^{*} 51$, $H L A-D R B 1^{*} 15$ and $\left.H L A-D Q B 1^{*} 06\right)$ and susceptibility to SLE in the Saudi population (5). In African-American women, a singlenucleotide polymorphism (SNP) which is closely associated with SLE, rs9271366, was found near the HLA-DRB1 gene (6).

Although genetic variations within the MHC are associated with the development of SLE, its role in the development of the clinical manifestations and autoantibody production has not been well defined. A meta-analysis of 4 independent European SLE case collections was previously performed in an effort to identify associations between SLE sub-phenotypes and MHC SNP genotypes, HLA alleles, and variant HLA amino acids. The results provided strong evidence for a multilevel risk model for $H L A-D R B 1^{*}$ 03:01 in SLE, wherein the association with anti-Ro and anti-La antibody-positive SLE is much stronger than with SLE without these autoantibodies (7). Despite the research which has been performed to date, a complete picture of these 
correlations has not yet been painted, and further studies are still needed to shed light on the associations between SLE and its susceptibility genes. In addition, novel methods should be used to investigate this subject.

Using a predictive bioinformatics algorithm, in a previous study, Mantila Roosa et al created a linear model of gene expression and identified 44 transcription factor-binding motifs and 29 miRNA-binding sites that were predicted to regulate gene expression across a time course. In addition to known sites, novel transcription factor-binding motifs and several novel miRNA-binding sites were identified throughout the time course. These time-dependent regulatory mechanisms may be important for controlling the loading-induced bone formation process (8). This integrated bioinformatics analysis method was also used in this study. Although the link between MHC and SLE has been proven, further investigations are likely to reveal the involvement of MHC in simple and complex genetic diseases, such as SLE. Indeed, we are interested in studying $\mathrm{MHC}, \mathrm{CpG}$ methylation and transcribed ultra-conserved region (T-UCR) as a first step toward a better understanding of the regulation of gene expression in SLE. In the present study, we provide an extensive view of SLE based on an integrated bioinformatics analysis of MHC, CpG methylation and T-UCR datasets.

\section{Materials and methods}

Patients and controls. Whole blood samples from 15 patients with SLE ( 8 females; 7 males; aged 18-50 years, with an average age of $35.64 \pm 11.27$ years) and 15 normal healthy controls ( 8 females; 7 males; aged $20-45$ years, with an average age of 33.47 \pm 9.61 years) were collected from the 181st Hospital of Guilin, China, between January and September 2011. The SLE diagnoses were confirmed based on pathological and clinical evidence according to the American Rheumatism Association classification criteria $(9,10)$. Written informed consent was obtained from all the subjects or their guardians. The use of biopsy material for studies beyond routine diagnosis was approved by the Ethics Committee of the 181st Hospital of Guilin. This study abides by the Helsinki Declaration on Ethical Principles for Medical Research Involving Human Subjects.

MHC gene capture, hMeDIP-chip and T-UCR microarray analysis. Genomic DNA was isolated from peripheral blood samples. According to the MHC genomic sequence, a completely complementary probe was designed and fixed on a support and then applied to the genomic DNA after coupling with a probe connector. The unhybridized probe was washed away, and the probe that had hybridized with the DNA was eluted to directly build a library for DNA sequencing (HiSeq 2000 high-throughput sequencing). The MHC region capture technology was based on the NimbleGen SeqCap EZ Choice Library, enabling the deep sequencing coverage of the human MHC region. The data were analyzed using the Chi-squared test with Yates' correction for continuity.

Genomic DNA was extracted using a DNeasy Blood and Tissue kit (Qiagen, Fremont, CA, USA). The sonicated genomic DNA $(1 \mu \mathrm{g})$ was used for immunoprecipitation with a mouse monoclonal antibody. For DNA labeling, a NimbleGen Dual-Color DNA Labeling kit was used according to the manufacturer's instructions detailed in the NimbleGen hMeDIP-chip protocol (NimbleGen Systems, Inc., Madison, WI, USA). The microarrays were hybridized in Nimblegen hybridization buffer/hybridization component $\mathrm{A}$ in a hybridization chamber (Hybridization System-Nimblegen Systems, Inc., Madison, WI, USA). For array hybridization, the NimbleGen Promoter plus CpG Island array (Roche, Basel, Switzerland) was used.

The Arraystar Human T-UCR Microarray profiles the expression of 1,518 long non-coding RNAs (lncRNAs) and 2,261 mRNAs with transcription units (TU) that overlap UCRs in either the sense or antisense orientation. Sample RNA labeling and array hybridization were performed according to the Agilent One-Color Microarray-Based Gene Expression Analysis protocol (Agilent Technology, Santa Clara, CA, USA), with minor modifications. The hybridized arrays were washed, fixed and scanned, using Agilent DNA Microarray Scanner (part no. G2505C). Agilent Feature Extraction software (version 11.0.1.1) was used to analyze the acquired array images. Quantile normalization and subsequent data processing were performed using the GeneSpring GX v12.1 software package (Agilent Technologies).

\section{Bioinformatics analysis}

CpG methylation enrichment in the MHC segment and analysis for differential enrichment. The MHC gene capture sequencing segment was chr6:28477797-33448354. To search the enrichment location, we analyzed the $\mathrm{CpG}$ peaks in the MHC segment.

T-UCR expression in the MHC segment. To search for the location of transcripts, we analyzed T-UCR expression in the MHC segment.

Effect of the CpG methylation level and T-UCR expression level in immunological processes. We analyzed all methylated CpGs, T-UCR, and their corresponding genes, and then analyzed the related genes with regard to immunological processes. To further examine the functions of these genes, we used the Online Gene Ontology Tool EASE (http://david. abcc.ncifcrf.gov/ease/ease1.htm). The differentially expressed genes were classified with regard to biological processes. Gene Ontology (GO) and KEGG pathway mapping of the genes was performed using the web-accessible DAVID annotation system.

Correlation of MHC mutation with CpG methylation. To identify correlations, we calculated the data of differential $\mathrm{CpG}$ methylation and MHC mutation and analyzed the correlation coefficients.

\section{Results}

Capturing the number of genes and SNP loci in the MHC region. We obtained 150 genes and 27,066 SNPs by MHC gene capture and high-throughput sequencing in the patients with SLE compared with the normal controls (data not shown).

hMeDIP-chip. The 3,826 genes with CpG islands had significantly different methylation levels in the patients with SLE compared with the normal controls, as was also previously noted (11).

T-UCR microarray analysis. To identify potential differentially expressed T-UCRs, we performed fold change filtering of 
Table I. Twelve CpG-methylated enrichment sites in the MHC segment.

\begin{tabular}{|c|c|c|c|c|c|}
\hline CpG name (hg19) & Length (bp) & Control & SLE & Gene name & Location \\
\hline chr6:30042918-30043500 & 582 & & 1 & $R N F 39$ & Promoter \\
\hline chr6:31323946-31325211 & 1,265 & & 1 & $H L A-B$ & Promoter \\
\hline chr6:31695894-31698245 & 2,351 & & 1 & $D D A H 2$ & Promoter \\
\hline chr6:31695894-31698245 & 2,351 & & 1 & $L Y 6 G 6 C$ & Promoter \\
\hline chr6:31695894-31698245 & 2,351 & & 1 & MSH5 & Promoter \\
\hline chr6:32935896-32936792 & 896 & & 1 & $B R D 2$ & Promoter \\
\hline chr6:29521110-29521833 & 723 & 1 & & $U B D$ & Intergenic \\
\hline chr6:30538983-30539487 & 504 & 1 & & $A B C F 1$ & Promoter \\
\hline chr6:30684836-30685503 & 667 & 1 & & $M D C 1$ & Promoter \\
\hline chr6:30684836-30685503 & 667 & 1 & & $T U B B$ & Promoter \\
\hline chr6:31548436-31549277 & 841 & 1 & & $L S T 1$ & Promoter \\
\hline chr6:32975684-32975926 & 242 & 1 & & $H L A-D P B 2$ & Intragenic \\
\hline
\end{tabular}

MHC, major histocompatibility complex; Control, healthy control subjects; SLE, systemic lupus erythematosus patients.

Table II. HLA-B promoter region and $H L A-D P B 2$ intragenic region in patients with SLE.

$H L A-B$ promoter region (chr6:31323946-31325211, 1265 bp)

CGAAGTCCCAGGTCCCGGACGGGGCTCTCAGGGTCTCAGGCTCCGAGGGCCGCGTCTGCAATGGGGAGGCGCAG CGTTGGGGATTCCCCACTCCCCTGAGTTTCACTTCTTCTCCCAACTTGTGTCGGGTCCTTCTTCCAGGATACTCGTG ACGCGTCCCCACTTCCCACTCCCATTGGGTATTGGATATCTAGAGAAGCCAATCAGCGTCGCCGCGGTCCCAGTTC TAAAGTCCCCACGCACCCACCCGGACTCAGAGTCTCCTCAGACGCCGAGATGCTGGTCATGGCGCCCCGAACCGT CCTCCTGCTGCTCTCGGCGGCCCTGGCCCTGACCGAGACCTGGGCCGGTGAGTGCGGGTCGGGAGGGAAATGGC CTCTGCCGGGAGGAGCGAGGGGACCGCAGGCGGGGGCGCAGGACCTGAGGAGCCGCGCCGGGAGGAGGGTCGG GCGGGTCTCAGCCCCTCCTCACCCCCAGGCTCCCACTCCATGAGGTATTTCTACACCTCCGTGTCCCGGCCCGGCC GCGGGGAGCCCCGCTTCATCTCAGTGGGCTACGTGGACGACACCCAGTTCGTGAGGTTCGACAGCGACGCCGCG AGTCCGAGAGAGGAGCCGCGGGCGCCGTGGATAGAGCAGGAGGGGCCGGAGTATTGGGACCGGAACACACAGA TCTACAAGGCCCAGGCACAGACTGACCGAGAGAGCCTGCGGAACCTGCGCGGCTACTACAACCAGAGCGAGGCC GGTGAGTGACCCCGGCCCGGGGCGCAGGTCACGACTCCCCATCCCCCACGTACGGCCCGGGTCGCCCCGAGTCTC CGGGTCCGAGATCCGCCTCCCTGAGGCCGCGGGACCCGCCCAGACCCTCGACCGGCGAGAGCCCCAGGCGCGTT TACCCGGTTTCATTTTCAGTTGAGGCCAAAATCCCCGCGGGTTGGTCGGGGCGGGGCGGGGCTCGGGGGACTGGG CTGACCGCGGGGCCGGGGCCAGGGTCTCACACCCTCCAGAGCATGTACGGCTGCGACGTGGGGCCGGACGGGCG CCTCCTCCGCGGGCATGACCAGTACGCCTACGACGGCAAGGATTACATCGCCCTGAACGAGGACCTGCGCTCCTG GACCGCCGCGGACACGGCGGCTCAGATCACCCAGCGCAAGTGGGAGGCGGCCCGTGAGGCGGAGCAGCGGAGA GCCTACCTGGAGGGCGAGTGCGTGGAGTGGCTCCGCAGATACCTGGAGAACGGGAAGGACAAGCTGGAGCGCGC HLA-DPB2 intragenic region (chr6:32975684-32975926, 242 bp)

CGAGGCCGTGTGGCGTCTGCCTGAGTTTGGTGACTTTGCCCGCTTTGACCCGCAGGGCGGGCTGGCCGGCATCGC CGCAATCAAAGCCCATCTGGACATCCTGGTGGAGCGCTCCAACCGCAGCAGAGCCATCAACGGTACCGGCCCTCC CTCTGCCCACCCAGTCAGGCGGGAAGGTCCAGAGAAACTTCCTCCCAGTTCCTAGGCTCCCATCACTCTGGGGCG CGCTCTCAGCGCCCGCGC

SLE, systemic lupus erythematosus; HLA, human leukocyte antigen.

the SLE patients compared with the normal controls. We found a signature of 8 upregulated T-UCRs and 29 downregulated T-UCRs (data not shown).

$C p G$ peak in the MHC segment. To search for enrichment locations, we analyzed $\mathrm{CpG}$ peaks in the MHC segment. The results indicated the enrichment of $12 \mathrm{CpG}$-methylated sites (Table I), with 6 in the patients with SLE and 6 in the normal controls. One $\mathrm{CpG}$-methylated enrichment site was found to be located in the $H L A-B$ promoter region (chr6:31323946-31325211, 1,265 bp) (Table II) in the patients with SLE; another site was located in the HLA-DPB2 intragenic region (chr6:32975684-32975926, 242 bp) (Table II) in the control group. 


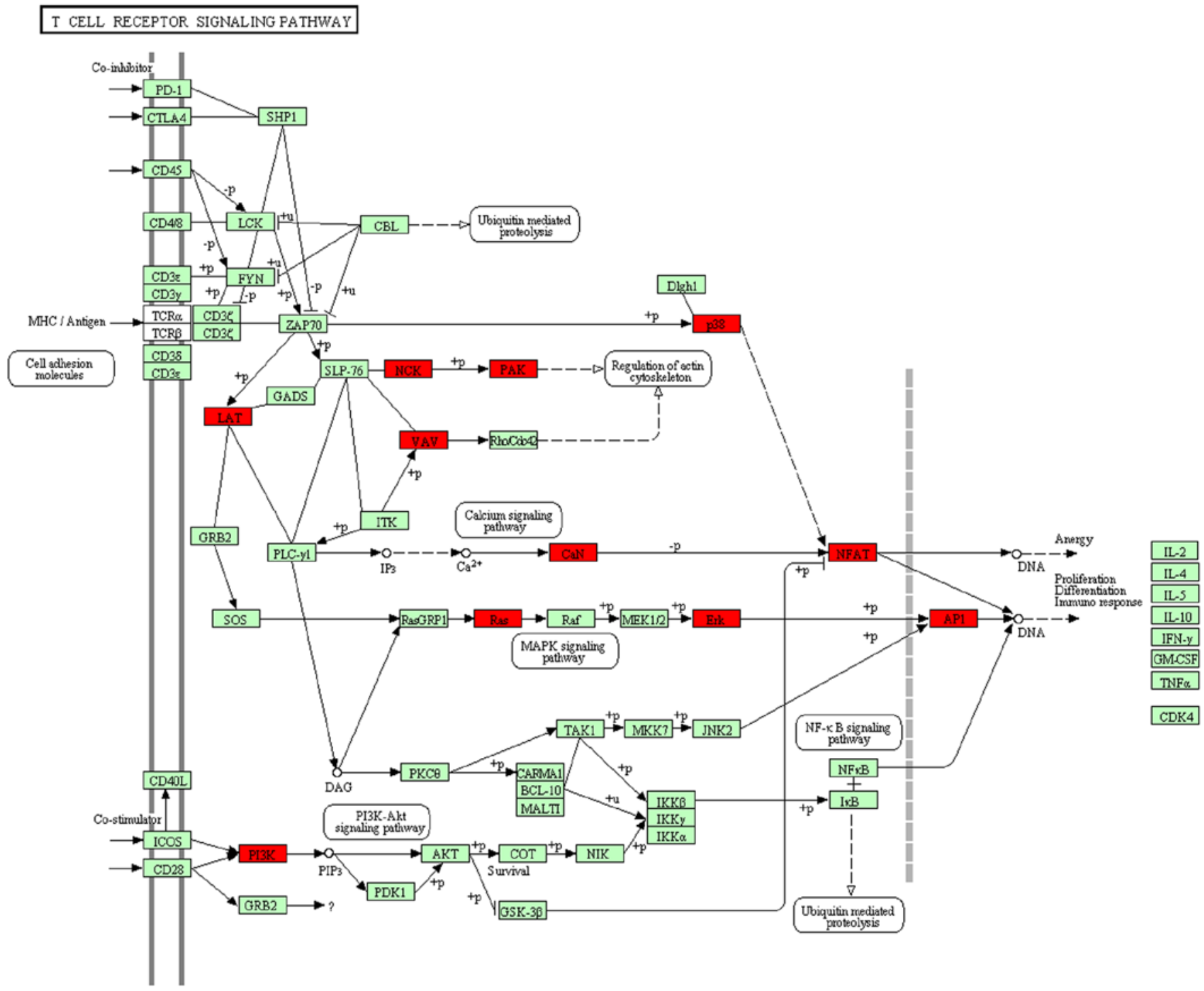

Figure 1. The immune-correlated process KEGG pathway hsa04660 $\mathrm{T}$ cell receptor signaling pathway.

$T$-UCR expression in the MHC segment. In the present study, we analyzed the expression of all T-UCRs; however, UCR-overlapping and UCR-proximal genes were not discovered in the MHC segment.

Effect of the CpG methylation level on immunological processes. We annotated corresponding CpG-methylated genes in the patients with SLE with GO schemes using the DAVID gene annotation tool. The genes produced a total of 97 GO terms in the patients with SLE (Table III). However, no significant enrichment was found for immune-correlated process GO terms, such as GO:0006955 - immune response (48 genes: TNFAIP8 L2, ITGAL, GALNT2, YWHAZ, LST1, TOLLIP, IFITM2, IFITM3, SUSD2, TLR2, NLRX1, VTN, TLR5, CX3CL1, PYDC1, FTH1, IGF1R, TUBB, IL2RG, CFD, SPON2, APLN, SPN, DNAJA3, POLL, TRPM4, DBNL, IL18R1, SMAD6, EOMES, CNPY3, STXBP2, POLR3A, HLA-B, VAV1, WAS, CDID, LAT, CYBA, PRELID1, GPI, SARM1, ULBP1, TGFBR3, ADAM17, TCF12, ICOSLG, TNFAIP1, $\mathrm{P}=0.999999$, FDR >0.05).
In addition, we obtained 3 KEGG pathways for genes in patients with SLE (Table IV), although no significant enrichment was found for immune-correlated process KEGG pathways, such as hsa04660:T cell receptor signaling pathway (Fig. 1; 15 genes: PIK3CG, HRAS, VAV1, LOC407835, MAPK1, LAT, MAPK12, NCK1, PAK4, JUN, NFAT5, PPP3CB, CHP, PIK3R3, NFATC1, $\mathrm{P}=0.406339$, FDR >0.05).

Effect of T-UCR expression levels on immunological processes. We also annotated T-UCR-corresponding genes with GO schemes using the DAVID gene annotation tool. The genes produced total $43 \mathrm{GO}$ terms in patients with SLE (Table V); however, no significant enrichment was found for immune-correlated process GO terms. In addition, we did not obtain immune-correlated process KEGG pathways for the genes in patients with SLE, i.e., there was no significant enrichment.

Correlation between MHC mutation and $\mathrm{CpG}$ methylation. In this study, we found 4SNPs in the CpG promoter(chr6:31323946- 
Table III. GO term annotations of corresponding CpG-methylated genes in SLE patients.

\begin{tabular}{|c|c|c|c|}
\hline GO term & $\begin{array}{l}\text { Gene } \\
\text { count }\end{array}$ & P-value & FDR \\
\hline GO:0030182 - neuron differentiation & 113 & $1.39 \mathrm{E}-13$ & $2.58 \mathrm{E}-10$ \\
\hline GO:0051252 - regulation of RNA metabolic process & 319 & 2.37E-10 & $4.41 \mathrm{E}-07$ \\
\hline GO:0006355 - regulation of transcription, DNA-dependent & 313 & $2.50 \mathrm{E}-10$ & $4.66 \mathrm{E}-07$ \\
\hline GO:0045449 - regulation of transcription & 429 & $8.22 \mathrm{E}-10$ & $1.53 \mathrm{E}-06$ \\
\hline GO:0000904 - cell morphogenesis involved in differentiation & 66 & $3.46 \mathrm{E}-09$ & $6.45 \mathrm{E}-06$ \\
\hline GO:0048666 - neuron development & 82 & $1.08 \mathrm{E}-08$ & $2.01 \mathrm{E}-05$ \\
\hline GO:0007409 - axonogenesis & 55 & $1.18 \mathrm{E}-08$ & 2.19E-05 \\
\hline GO:0006350 - transcription & 349 & $2.66 \mathrm{E}-08$ & $4.96 \mathrm{E}-05$ \\
\hline GO:0048667 - cell morphogenesis involved in neuron differentiation & 57 & $3.36 \mathrm{E}-08$ & $6.26 \mathrm{E}-05$ \\
\hline GO:0000902 - cell morphogenesis & 83 & $5.01 \mathrm{E}-08$ & $9.34 \mathrm{E}-05$ \\
\hline GO:0007389 - pattern specification process & 66 & $1.46 \mathrm{E}-07$ & $2.73 \mathrm{E}-04$ \\
\hline GO:0048812 - neuron projection morphogenesis & 56 & $1.71 \mathrm{E}-07$ & $3.18 \mathrm{E}-04$ \\
\hline GO:0031175 - neuron projection development & 63 & $3.43 \mathrm{E}-07$ & $6.39 \mathrm{E}-04$ \\
\hline GO:0048858 - cell projection morphogenesis & 61 & $3.52 \mathrm{E}-07$ & $6.56 \mathrm{E}-04$ \\
\hline GO:0032989 - cellular component morphogenesis & 87 & $4.22 \mathrm{E}-07$ & $7.86 \mathrm{E}-04$ \\
\hline GO:0032990 - cell part morphogenesis & 62 & $7.71 \mathrm{E}-07$ & 0.001437153 \\
\hline GO:0006357 - regulation of transcription from RNA polymerase II promoter & 138 & $1.20 \mathrm{E}-06$ & 0.002241345 \\
\hline GO:0030030 - cell projection organization & 80 & $1.79 \mathrm{E}-06$ & 0.003344582 \\
\hline GO:0003002 - regionalization & 50 & $2.55 \mathrm{E}-06$ & 0.004748657 \\
\hline GO:0021953 - central nervous system neuron differentiation & 18 & 2.92E-06 & 0.005449045 \\
\hline GO:0030900 - forebrain development & 41 & 4.82E-06 & 0.008984544 \\
\hline GO:0048663 - neuron fate commitment & 18 & $6.52 \mathrm{E}-06$ & 0.012150091 \\
\hline GO:0048598 - embryonic morphogenesis & 67 & $1.19 \mathrm{E}-05$ & 0.022252245 \\
\hline GO:0021954 - central nervous system neuron development & 15 & $1.47 \mathrm{E}-05$ & 0.02743134 \\
\hline GO:0045944 - positive regulation of transcription from RNA polymerase II promoter & 77 & $1.68 \mathrm{E}-05$ & 0.031223255 \\
\hline GO:0007411 - axon guidance & 31 & $1.93 \mathrm{E}-05$ & 0.035915973 \\
\hline GO:0045165 - cell fate commitment & 37 & $2.06 \mathrm{E}-05$ & 0.038453597 \\
\hline GO:0021872 - generation of neurons in the forebrain & 10 & $2.17 \mathrm{E}-05$ & 0.040470383 \\
\hline GO:0045893 - positive regulation of transcription, DNA-dependent & 93 & $2.77 \mathrm{E}-05$ & 0.051580919 \\
\hline GO:0051254 - positive regulation of RNA metabolic process & 93 & $3.85 \mathrm{E}-05$ & 0.071644433 \\
\hline GO:0006928 - cell motion & 92 & $3.98 \mathrm{E}-05$ & 0.074087062 \\
\hline GO:0007423 - sensory organ development & 52 & 4.33E-05 & 0.08062919 \\
\hline GO:0007169 - transmembrane receptor protein tyrosine kinase signaling pathway & 51 & 4.81E-05 & 0.089665676 \\
\hline GO:0021879 - forebrain neuron differentiation & 9 & 4.99E-05 & 0.092920613 \\
\hline $\begin{array}{l}\text { GO:0045935 - positive regulation of nucleobase, nucleoside, nucleotide } \\
\text { and nucleic acid metabolic process }\end{array}$ & 114 & $6.21 \mathrm{E}-05$ & 0.115713935 \\
\hline GO:0051173 - positive regulation of nitrogen compound metabolic process & 117 & $6.28 \mathrm{E}-05$ & 0.116921469 \\
\hline GO:0060284 - regulation of cell development & 47 & $8.31 \mathrm{E}-05$ & 0.154746845 \\
\hline GO:0045941 - positive regulation of transcription & 104 & $9.33 \mathrm{E}-05$ & 0.173822702 \\
\hline GO:0050767 - regulation of neurogenesis & 40 & $1.00 \mathrm{E}-04$ & 0.186897834 \\
\hline GO:0045664 - regulation of neuron differentiation & 34 & $1.11 \mathrm{E}-04$ & 0.206978981 \\
\hline GO:0010628 - positive regulation of gene expression & 106 & $1.20 \mathrm{E}-04$ & 0.223869384 \\
\hline GO:0010557 - positive regulation of macromolecule biosynthetic process & 117 & $1.23 \mathrm{E}-04$ & 0.228227922 \\
\hline GO:0016192 - vesicle-mediated transport & 105 & $1.36 \mathrm{E}-04$ & 0.253359896 \\
\hline GO:0051960 - regulation of nervous system development & 44 & $1.46 \mathrm{E}-04$ & 0.271915907 \\
\hline GO:0031328 - positive regulation of cellular biosynthetic process & 121 & $1.62 \mathrm{E}-04$ & 0.302155781 \\
\hline GO:0009891 - positive regulation of biosynthetic process & 122 & $1.98 \mathrm{E}-04$ & 0.368213146 \\
\hline GO:0007167 - enzyme linked receptor protein signaling pathway & 68 & $2.08 \mathrm{E}-04$ & 0.386237807 \\
\hline GO:0045892 - negative regulation of transcription, DNA-dependent & 70 & 2.33E-04 & 0.432663991 \\
\hline GO:0030817 - regulation of cAMP biosynthetic process & 27 & $3.08 \mathrm{E}-04$ & 0.572491129 \\
\hline GO:0002009 - morphogenesis of an epithelium & 27 & $3.08 \mathrm{E}-04$ & 0.572491129 \\
\hline
\end{tabular}


Table III. Continued.

\begin{tabular}{|c|c|c|c|}
\hline GO term & $\begin{array}{l}\text { Gene } \\
\text { count }\end{array}$ & P-value & FDR \\
\hline GO:0030902 - hindbrain development & 19 & $3.48 \mathrm{E}-04$ & 0.647196595 \\
\hline GO:0021537 - telencephalon development & 20 & $3.49 \mathrm{E}-04$ & 0.648170377 \\
\hline GO:0021761 - limbic system development & 13 & $3.51 \mathrm{E}-04$ & 0.6529104 \\
\hline GO:0051253 - negative regulation of RNA metabolic process & 70 & $3.83 \mathrm{E}-04$ & 0.7106764 \\
\hline $\begin{array}{l}\text { GO:0045934 - negative regulation of nucleobase, nucleoside, nucleotide } \\
\text { and nucleic acid metabolic process }\end{array}$ & 93 & $3.88 \mathrm{E}-04$ & 0.7213528 \\
\hline GO:0010604 - positive regulation of macromolecule metabolic process & 144 & $3.97 \mathrm{E}-04$ & 0.737772867 \\
\hline GO:0030814 - regulation of cAMP metabolic process & 27 & 4.29E-04 & 0.795879925 \\
\hline GO:0009792 - embryonic development ending in birth or egg hatching & 65 & $5.31 \mathrm{E}-04$ & 0.984899092 \\
\hline GO:0008285 - negative regulation of cell proliferation & 69 & $5.90 \mathrm{E}-04$ & 1.094286156 \\
\hline GO:0051172 - negative regulation of nitrogen compound metabolic process & 93 & $6.13 \mathrm{E}-04$ & 1.136642775 \\
\hline GO:0009890 - negative regulation of biosynthetic process & 101 & $6.30 \mathrm{E}-04$ & 1.168027793 \\
\hline GO:0010558 - negative regulation of macromolecule biosynthetic process & 97 & $6.65 \mathrm{E}-04$ & 1.231652838 \\
\hline GO:0009952 - anterior/posterior pattern formation & 33 & $6.76 \mathrm{E}-04$ & 1.252734624 \\
\hline GO:0031327 - negative regulation of cellular biosynthetic process & 99 & $6.87 \mathrm{E}-04$ & 1.273119736 \\
\hline GO:0043583 - ear development & 25 & $6.92 \mathrm{E}-04$ & 1.282237143 \\
\hline GO:0051339 - regulation of lyase activity & 26 & 7.49E-04 & 1.385942497 \\
\hline GO:0048732 - gland development & 32 & 7.50E-04 & 1.387778635 \\
\hline GO:0045761 - regulation of adenylate cyclase activity & 25 & $8.13 \mathrm{E}-04$ & 1.504562886 \\
\hline GO:0022037 - metencephalon development & 13 & $9.00 \mathrm{E}-04$ & 1.663443567 \\
\hline GO:0048568 - embryonic organ development & 38 & $9.18 \mathrm{E}-04$ & 1.697487359 \\
\hline GO:0021766 - hippocampus development & 10 & 9.31E-04 & 1.721218707 \\
\hline GO:0016481 - negative regulation of transcription & 83 & 9.44E-04 & 1.744715102 \\
\hline GO:0030808 - regulation of nucleotide biosynthetic process & 27 & 0.001237 & 2.280679952 \\
\hline GO:0030802 - regulation of cyclic nucleotide biosynthetic process & 27 & 0.001237 & 2.280679952 \\
\hline GO:0010629 - negative regulation of gene expression & 89 & 0.001288 & 2.373367738 \\
\hline GO:0031279 - regulation of cyclase activity & 25 & 0.001292 & 2.380392647 \\
\hline GO:0035107 - appendage morphogenesis & 25 & 0.001292 & 2.380392647 \\
\hline GO:0035108 - limb morphogenesis & 25 & 0.001292 & 2.380392647 \\
\hline GO:0021543 - pallium development & 15 & 0.00132 & 2.432197518 \\
\hline GO:0014031 - mesenchymal cell development & 16 & 0.001327 & 2.444625723 \\
\hline GO:0048762 - mesenchymal cell differentiation & 16 & 0.001327 & 2.444625723 \\
\hline GO:0060562 - epithelial tube morphogenesis & 19 & 0.001468 & 2.701431115 \\
\hline GO:0010941 - regulation of cell death & 134 & 0.001518 & 2.791424456 \\
\hline GO:0035295 - tube development & 45 & 0.001562 & 2.870764354 \\
\hline GO:0048705 - skeletal system morphogenesis & 27 & 0.001632 & 2.999117627 \\
\hline GO:0060485 - mesenchyme development & 16 & 0.001648 & 3.027543666 \\
\hline GO:0030799 - regulation of cyclic nucleotide metabolic process & 27 & 0.001868 & 3.424511611 \\
\hline GO:0001709 - cell fate determination & 12 & 0.001898 & 3.478414435 \\
\hline GO:0043009 - chordate embryonic development & 62 & 0.001904 & 3.49042025 \\
\hline GO:0016331 - morphogenesis of embryonic epithelium & 17 & 0.001959 & 3.588514741 \\
\hline GO:0042127 - regulation of cell proliferation & 129 & 0.002101 & 3.843962583 \\
\hline GO:0048736 - appendage development & 25 & 0.002293 & 4.187971146 \\
\hline GO:0060173 - limb development & 25 & 0.002293 & 4.187971146 \\
\hline GO:0051349 - positive regulation of lyase activity & 17 & 0.002374 & 4.334185806 \\
\hline GO:0035239 - tube morphogenesis & 29 & 0.002504 & 4.564895517 \\
\hline GO:0043067 - regulation of programmed cell death & 132 & 0.002508 & 4.573381853 \\
\hline GO:0017145 - stem cell division & 6 & 0.002742 & 4.98879198 \\
\hline
\end{tabular}

A P-value $<0.005$ was considered to indicate a statistically significant difference. The false discovery rate (FDR) of a set of predictions is the expected percentage of false predictions in the set of predictions; an FDR $<5 \%$ may be quite meaningful. SLE, systemic lupus erythematosus. 
Table IV. KEGG pathway annotation of corresponding CpG-methylated genes in patients with SLE.

\begin{tabular}{lccr}
\hline Pathways & Gene count & P-value & FDR \\
\hline hsa05200: pathways in cancer & 60 & $5.25 \mathrm{E}-04$ & 0.6471034 \\
hsa04916: melanogenesis & 23 & 0.002387 & 2.9086362 \\
hsa05217: basal cell carcinoma & 15 & 0.003991 & 4.8195711 \\
\hline
\end{tabular}

A P-value $<0.005$ was considered to indicate a statistically significant difference. The false discovery rate (FDR) of a set of predictions is the expected percentage of false predictions in the set of predictions; an FDR $<5 \%$ may be quite meaningful. SLE, systemic lupus erythematosus.

31325211) of $H L A-B$ and 2 SNPs in chr6:29521110-29521833 in the control patients (Table VI).

\section{Discussion}

The first genetic factors to be identified as important in the pathogenesis of SLE were those of the MHC on chromosome 6. It is now widely accepted that MHC genes constitute a part of the genetic susceptibility to SLE (12). However, previous studies on SLE have lacked statistical power and the genetic resolution to fully define the influences of the MHC $(13,14)$. In this study, we attempted to identify MHC, CpG methylation and T-UCR to reveal the potential mechanisms responsible for the development of SLE using a novel and combinatorial approach involving MHC gene capture technology, hMeDIPchip, T-UCR microarray and bioinformatics analysis. A total of 27,066 SNPs were detected and thus these may be involved in SLE. Moreover, we integrated the datasets and identified 6 of the most important SNPs in SLE. Our next step is to perform research on the function of these SNPs.

HLA antigens and genes have long been reported to be associated with SLE susceptibility in a number of populations (15). With advances in technologies, such as genome-wide association studies, a number of newly discovered SLE-associated SNPs have been reported in recent years. These include HLA-DRB1/HLA-DQA1 rs9271366 and $H L A-D Q B 1 / H L A-D Q A 2$ rs9275328 (15). Previously, a meta-analysis of the MHC region in patients with SLE was performed to determine associations with both SNPs and classical HLA alleles. The results of a conditional analysis and model choice with the use of the Bayesian information criterion indicated that the best model for SLE association includes both classical loci (HLA-DRB1*03:01, HLA-DRB1*08:01 and $H L A-D Q A 1^{*} 01: 02$ ) and 2 SNPs, rs8192591 (in class III and upstream of NOTCH4) and rs2246618 (MICB in class I) (16). Single-marker analyses have revealed strong signals for SNPs within several MHC regions, as well as for $H L A-D R B 1$. The most strongly associated DRB1 alleles are *0301, *1401 and *1501, and the MHC region SNP demonstrating the strongest evidence of an association with SLE is rs3117103 (3). These results delineate with high resolution several $\mathrm{MHC}$ regions which contribute independently to the risk of developing SLE. In the present study, we integrated the MHC and CpG-methylated datasets, and the results indicated $\mathrm{CpG}$ methylation enrichment at 6 sites: RNF39, HLA-B, DDAH2, LY6G6C, MSH5 and $B R D 2$ in the $\mathrm{MHC}$ regions of SLE. These genes play important roles in various immune diseases, including SLE. SNPs in the region of the RNF39 gene have been found to be associated with the disease course of HIV-1 $(17,18)$. Behcet's disease is a chronic inflammatory autoimmune disease that is strongly associated with $H L A-B 51$ and $-A 26$. It has previously been suggested that RNF39 is involved in the etiology of Behcet's disease (19). The MHC region is suspected to host susceptibility loci for HIV-related Kaposi's sarcoma, involving the rs1065356 (LY6G6C) and rs3749953 (MSH5-SAPCD1) (20). MSH5 has been found to be mutated in patients with common variable immunodeficiency (21). In a previous study, a significant increase in the frequency of $H L A-A^{*} 01, A^{*} 03, A^{*} 11, A^{*} 23$, $A^{*} 26 A^{*} 69, H L A-B^{*} 27, B^{*} 40, B^{*} 49, B^{*} 51, B^{*} 52, B^{*} 53, B^{*} 54, B^{*} 95$, HLA-DRBI 01 , DRBI 03, DRBI 11 and DRBI 14 was observed in SLE patients, indicating a positive association of these alleles with SLE. By contrast, $H L A-A^{*} 24, A^{*} 29, A^{*} 31, A^{*} 34$, $A^{*} 68, A^{*} 92, H L A-B^{*} 18$ and $H L A-D R B 1^{*} 12$ were found to be decreased in the patient group compared to the controls, indicating a negative association of these alleles with SLE. Thus, it was concluded that SLE is associated with certain MHC alleles, such as $H L A-B$, in the Pakistani population (12). As the results of the present study indicated the enrichment of one $\mathrm{CpG}$ methylation site located in the $H L A-B$ promoter region, $H L A-B$ may indeed play an important role in the pathogenesis of SLE. Moreover, we found 4 SNPs (rs1050683, rs12697943, rs17881210 and rs1065378) in the CpG region of the $H L A-B$ promoter and 2 SNPs (rs17184255 and rs16895070) in MHC regions. These SNPs were significantly associated with an increased risk of developing SLE.

SLE is an autoimmune disease with known genetic, epigenetic, and environmental risk factors. Epigenetic events play a central role in the priming, differentiation and subset determination of $\mathrm{T}$ lymphocytes. CpG-DNA methylation and post-translational modifications to histone tails are the two most well-accepted epigenetic mechanisms. Furthermore, the involvement of epigenetic mechanisms in the pathogenesis of SLE has been suggested by the development of lupus-like symptoms in individuals who are treated with procainamide or hydralazine, resulting in a reduction in CpG-DNA methylation (22). In SLE, global CpG-DNA hypomethylation correlates with disease activity. A number of cytokine genes are overexpressed in $\mathrm{CD}^{+} \mathrm{T}$ lymphocytes from patients with SLE in a chromatin-dependent manner, including IL-6 (23). Regionspecific histone acetylation in certain tissues is associated with increased disease activity, whereas histone acetylation in other regions has protective effects. In SLE, acetylation of the TNF 
Table V. GO term annotation of T-UCR corresponding genes in SLE patients.

\begin{tabular}{|c|c|c|c|}
\hline GO term & $\begin{array}{l}\text { Gene } \\
\text { count }\end{array}$ & P-value & FDR \\
\hline GO:0008380 - RNA splicing & 35 & $1.24 \mathrm{E}-11$ & $2.15 \mathrm{E}-08$ \\
\hline GO:0006397 - mRNA processing & 37 & $2.04 \mathrm{E}-11$ & $3.55 \mathrm{E}-08$ \\
\hline GO:0016071 - mRNA metabolic process & 38 & $3.00 \mathrm{E}-10$ & $5.22 \mathrm{E}-07$ \\
\hline GO:0006396 - RNA processing & 47 & $6.45 \mathrm{E}-10$ & $1.12 \mathrm{E}-06$ \\
\hline GO:0006357 - regulation of transcription from RNA polymerase II promoter & 55 & $1.75 \mathrm{E}-09$ & $3.04 \mathrm{E}-06$ \\
\hline GO:0045449 - regulation of transcription & 129 & $1.15 \mathrm{E}-08$ & $1.99 \mathrm{E}-05$ \\
\hline GO:0051252 - regulation of RNA metabolic process & 97 & 5.09E-08 & $8.85 \mathrm{E}-05$ \\
\hline $\begin{array}{l}\text { GO:0045935 - positive regulation of nucleobase, nucleoside, } \\
\text { nucleotide and nucleic acid metabolic process }\end{array}$ & 44 & $7.25 \mathrm{E}-07$ & 0.001261 \\
\hline GO:0051254 - positive regulation of RNA metabolic process & 37 & $9.25 \mathrm{E}-07$ & 0.00161 \\
\hline GO:0006355 - regulation of transcription, DNA-dependent & 91 & $1.01 \mathrm{E}-06$ & 0.00175 \\
\hline GO:0010558 - negative regulation of macromolecule biosynthetic process & 40 & $1.07 \mathrm{E}-06$ & 0.001865 \\
\hline GO:0000398 - nuclear mRNA splicing, via spliceosome & 19 & $1.20 \mathrm{E}-06$ & 0.002082 \\
\hline $\begin{array}{l}\text { GO:0000377 - RNA splicing, via transesterification reactions with bulged } \\
\text { adenosine as nucleophile }\end{array}$ & 19 & $1.20 \mathrm{E}-06$ & 0.002082 \\
\hline GO:0000375 - RNA splicing, via transesterification reactions & 19 & $1.20 \mathrm{E}-06$ & 0.002082 \\
\hline GO:0045944 - positive regulation of transcription from RNA polymerase II promoter & 31 & $1.60 \mathrm{E}-06$ & 0.002782 \\
\hline GO:0051173 - positive regulation of nitrogen compound metabolic process & 44 & $1.68 \mathrm{E}-06$ & 0.002914 \\
\hline GO:0031327 - negative regulation of cellular biosynthetic process & 40 & $1.97 \mathrm{E}-06$ & 0.003434 \\
\hline GO:0045893 - positive regulation of transcription, DNA-dependent & 36 & $2.06 \mathrm{E}-06$ & 0.003575 \\
\hline GO:0045941 - positive regulation of transcription & 40 & $2.26 \mathrm{E}-06$ & 0.00393 \\
\hline GO:0009890 - negative regulation of biosynthetic process & 40 & 3.34E-06 & 0.005812 \\
\hline GO:0048598 - embryonic morphogenesis & 27 & $3.46 \mathrm{E}-06$ & 0.006015 \\
\hline $\begin{array}{l}\text { GO:0045934 - negative regulation of nucleobase, nucleoside, nucleotide } \\
\text { and nucleic acid metabolic process }\end{array}$ & 37 & $3.86 \mathrm{E}-06$ & 0.00672 \\
\hline GO:0006350 - transcription & 101 & 4.00E-06 & 0.006958 \\
\hline GO:0010628 - positive regulation of gene expression & 40 & 4.62E-06 & 0.008041 \\
\hline GO:0051172 - negative regulation of nitrogen compound metabolic process & 37 & $5.24 \mathrm{E}-06$ & 0.009119 \\
\hline GO:0031328 - positive regulation of cellular biosynthetic process & 44 & 8.06E-06 & 0.014026 \\
\hline GO:0051253 - negative regulation of RNA metabolic process & 29 & 8.35E-06 & 0.014517 \\
\hline GO:0010605 - negative regulation of macromolecule metabolic process & 46 & 8.87E-06 & 0.015419 \\
\hline GO:0010604 - positive regulation of macromolecule metabolic process & 51 & $1.09 \mathrm{E}-05$ & 0.018888 \\
\hline GO:0009891 - positive regulation of biosynthetic process & 44 & $1.15 \mathrm{E}-05$ & 0.020058 \\
\hline GO:0016481 - negative regulation of transcription & 33 & $1.61 \mathrm{E}-05$ & 0.027966 \\
\hline GO:0045892 - negative regulation of transcription, DNA-dependent & 28 & $1.72 \mathrm{E}-05$ & 0.029862 \\
\hline GO:0010557 - positive regulation of macromolecule biosynthetic process & 41 & $3.08 \mathrm{E}-05$ & 0.053493 \\
\hline GO:0010629 - negative regulation of gene expression & 34 & $4.16 \mathrm{E}-05$ & 0.072327 \\
\hline GO:0000122 - negative regulation of transcription from RNA polymerase II promoter & 21 & $2.37 \mathrm{E}-04$ & 0.412261 \\
\hline GO:0048568 - embryonic organ development & 16 & $2.97 \mathrm{E}-04$ & 0.515968 \\
\hline GO:0016055 - Wnt receptor signaling pathway & 13 & 8.69E-04 & 1.501407 \\
\hline GO:0030900 - forebrain development & 14 & 8.89E-04 & 1.535269 \\
\hline GO:0043009 - chordate embryonic development & 22 & 0.001544 & 2.651627 \\
\hline GO:0009792 - embryonic development ending in birth or egg hatching & 22 & 0.001718 & 2.946609 \\
\hline GO:0046907 - intracellular transport & 35 & 0.002355 & 4.018647 \\
\hline GO:0015931 - nucleobase, nucleoside, nucleotide and nucleic acid transport & 11 & 0.002648 & 4.507588 \\
\hline GO:0048562 - embryonic organ morphogenesis & 12 & 0.002821 & 4.795051 \\
\hline
\end{tabular}

A P-value $<0.005$ was considered significant. The false discovery rate (FDR) of a set of predictions is the expected percent of false predictions in the set of predictions; an FDR $<5 \%$ may be quite meaningful. SLE, systemic lupus erythematosus; T-UCR, transcribed ultra-conserved regions. 
Table VI. Six CpG-methylated SNPs of the MHC segment in patients with SLE.

\begin{tabular}{llll}
$\begin{array}{l}\text { Chromosome } \\
\text { segment }\end{array}$ & \multicolumn{1}{c}{ SNP } & Gene & Location \\
\hline chr6:31324019 & rs1050683 & HLA-B & Exonic \\
chr6:31324057 & rs12697943 & HLA-B & Exonic \\
chr6:31324448 & rs17881210 & HLA-B & Intronic \\
chr6:31324633 & rs1065378 & HLA-B & Exonic \\
chr6:29521289 & rs17184255 & & Intergenic \\
chr6:29521557 & rs16895070 & & Intergenic \\
\hline
\end{tabular}

MHC, major histocompatibility complex; SLE, systemic lupus erythematosus; SNP, single nucleotidepolymorphism.

promoter in monocytes is associated with increased monocyte maturation and cytokine expression (24). Thus, a better understanding of the molecular events that contribute to epigenetic alterations and subsequent immune imbalance is essential for the establishment of disease biomarkers and the identification of potential therapeutic targets (22). To assess the role of DNA methylation in SLE, researchers collected $\mathrm{CD}^{+}{ }^{+} \mathrm{T}$-cells, $\mathrm{CD} 19^{+} \mathrm{B}$-cells, and $\mathrm{CD} 14^{+}$monocytes, and performed a genome-wide DNA methylation analysis with the use of IlluminaMethylation 450 microarrays. Interferon hypersensitivity was apparent in memory, naïve and regulatory T-cells, suggesting that this epigenetic state in lupus patients is established in progenitor cell populations. These cell type-specific effects are consistent with the disease-specific changes in the composition of the $\mathrm{CD} 4^{+}$population and suggest that shifts in the proportion of $\mathrm{CD}^{+}$subtypes can be monitored at $\mathrm{CpGs}$ with subtype-specific DNA methylation patterns (25). In the present study, we annotated the corresponding CpG-methylated genes using the DAVID gene annotation tool. However, immune-correlated process GO terms, such as GO:0006955immuneresponse, and KEGG pathways, such ashsa04660-T-cell receptor (TCR) signaling pathway exhibited no significant enrichment. The GO analysis did reveal that the 'theme' immune response (GO:0006955), which is known to be affected by anti-TNF treatment in the inflammatory tissue of rheumatoid arthritis patients, was significantly over-represented (26). Regardless, it is relevant to note in our context that our GO analysis identified immune functions as potentially relevant mechanisms. The activation of $\mathrm{T}$ lymphocytes is a key event for an efficient response of the immune system (hsa04660-TCR signaling pathway) and requires the involvement of the TCR as well as costimulatory molecules, such as CD28. The engagement of these receptors through interaction with a foreign antigen is associated with MHC molecules (27), and our findings may thus facilitate the selection of better target molecules for further studies. The findings of the present study may also aid future research by providing details of new pathways to be studied using a more focused approach, confirmation at the protein level and emphasis of the clinical significance.

lncRNAs are transcripts longer than $\sim 200$ nucleotides with little or no protein-coding capacity (28). Research has shown that lncRNAs play important roles in disease development and are associated with a number of human diseases, such as cancer, Alzheimer's disease and heart disease (29). T-UCR transcripts are a novel class of IncRNAs transcribed from UCRs, a class of 481 non-coding sequences located in both intra- and inter-genic regions of the genome. UCRs are absolutely conserved (100\%) between the orthologous regions of the human, rat, and mouse genomes and are actively transcribed $(30,31)$. It has recently been proven in cancer systems that differentially expressed T-UCRs alter the functional characteristics of malignant cells. Indeed, recent data suggest that T-UCRs are altered at the transcriptional level in human tumorigenesis and that the aberrant T-UCR expression profiles can be used to differentiate human cancer types $(31,32)$. Researchers observed that DNA hypomethylation induces T-UCR silencing in cancer cells, and the analysis of a large set of primary human tumors demonstrated that the hypermethylation of the described T-UCR CpG islands is a common event in the various tumor types (33). In the present study, we integrated the MHC and T-UCR datasets and examined the expression levels of T-UCR in the MHC segment by T-UCR microarray. We annotated the T-UCR corresponding genes using the DAVID gene annotation tool. However, no significant enrichment was found for immune-correlated process GO terms and KEGG pathways. Thus, T-UCR expression levels did not correlate with the commonly used clinicopathological features of the patients with SLE.

Taken together, in the present study, we identified 6 of the most important SNPs (rs1050683, rs12697943, rs17881210, rs1065378, rs17184255 and rs16895070) in patients with SLE. The present study indicates that SNPs in the MHC segment are potential biomarkers and are likely factors which are involved in the pathogenesis of SLE. However, further studies are required to investigate the mechanisms through which polymorphisms in this region lead to the development of SLE. A major advantage of combining multiple levels of measurement is the ability to dissect mechanisms not apparent in a single dimension. The integration of $\mathrm{MHC}, \mathrm{CpG}$ methylation, and T-UCR datasets is a powerful strategy for understanding SLE biology. Our findings provide insight into the potential contribution of anomalously regulated SNPs to the abnormalities in SLE and may aid in the structuring of antenatal diagnostic biomarkers of SLE, as well as in obtaining novel therapeutic targets which can be used in the treatment of patients with SLE. Moreover, our study of SNPs may aid in the development of novel methods which may prove to be useful for treating and preventing other diseases.

\section{Acknowledgements}

The authors of this study would like to thank the patients with SLE and the healthy volunteers who participated in this study. Bioinformatics analysis was performed by Shanghai Biotree Biotech Co., Ltd., Shanghai, China. The present study was supported financially by the Key Project of Guangxi Natural Science Foundation (no. 2012GXNSFDA053017), the Construction Project Planning Assignment of Guangxi Key Laboratory (no. 13-051-31) and the Scientific Problem Tackling of Guilin Science and Technology Program (no. 20130120-20), China. 


\section{References}

1. Mirkazemi S, Akbarian M, Jamshidi AR, Mansouri R, Ghoroghi S, Salimi Y, Tahmasebi Z and Mahmoudi M: Association of STAT4 rs7574865 with susceptibility to systemic lupus erythematosus in Iranian population. Inflammation 36: $1548-1552,2013$

2. Zhang J, Zhang Y, Yang J, Zhang L, Sun L, Pan HF, Hirankarn N, Ying D, Zeng S, Lee TL, et al: Three SNPs in chromosome $11 \mathrm{q} 23.3$ are independently associated with systemic lupus erythematosus in Asians. Hum Mol Genet 23: 524-533, 2014

3. Barcellos LF, May SL, Ramsay PP, Quach HL, Lane JA, Nititham J, Noble JA, Taylor KE, Quach DL, Chung SA, et al: High-density SNP screening of the major histocompatibility complex in systemic lupus erythematosus demonstrates strong evidence for independent susceptibility regions. PLoS Genet 5: e1000696, 2009.

4. Fernando MM, Freudenberg J, Lee A, Morris DL, Boteva L, Rhodes B, Gonzalez-Escribano MF, Lopez-Nevot MA, Navarra SV, Gregersen PK, Martin J; IMAGEN and Vyse TJ: Transancestral mapping of the $\mathrm{MHC}$ region in systemic lupus erythematosus identifies new independent and interacting loci at MSH5, HLA-DPB1 and HLA-G. Ann Rheum Dis 71: 777-784, 2012.

5. Al-Motwee S, Jawdat D, Jehani GS, Anazi H, Shubaili A, Sutton P, Uyar AF and Hajeer AH; AI-Motwee S: Association of HLA-DRB1*15 and HLADQB1*06 with SLE in Saudis. Ann Saudi Med 33: 229-234, 2013.

6. Ruiz-Narvaez EA, Fraser PA, Palmer JR, Cupples LA, Reich D, Wang YA, Rioux JD and Rosenberg L: MHC region and risk of systemic lupus erythematosus in African American women. Hum Genet 130: 807-815, 2011.

7. Morris DL, Fernando MM, Taylor KE, Chung SA, Nititham J, Alarcón-Riquelme ME, Barcellos LF, Behrens TW, Cotsapas C, Gaffney PM, et al; Systemic Lupus Erythematosus Genetics Consortium: MHC associations with clinical and autoantibody manifestations in European SLE. Genes Immun 15: 210-217, 2014.

8. Mantila Roosa SM, Turner CH and Liu Y: Regulatory mechanisms in bone following mechanical loading. Gene Regul Syst Bio 6: 43-53, 2012.

9. Tan EM, Cohen AS, Fries JF, Masi AT, McShane DJ, Rothfield NF, Schaller JG, Talal N and Winchester RJ: The 1982 revised criteria for the classification of systemic lupus erythematosus. Arthritis Rheum 25: 1271-1277, 1982.

10. Hochberg MC: Updating the American College of Rheumatology revised criteria for the classification of systemic lupus erythematosus. Arthritis Rheum 40: 1725, 1997.

11. Sui W, Tan Q, Yang M, Yan Q, Lin H, Ou M, Xue W, Chen J, Zou T, Jing H, et al: Genome-wide analysis of 5-hmC in the peripheral blood of systemic lupus erythematosus patients using an hMeDIP-chip. Int J Mol Med 35: 1467-1479, 2015.

12. Hussain N, Jaffery G, Sabri AN and Hasnain S: HLA association in SLE patients from Lahore-Pakistan. Bosn J Basic Med Sci 11: 20-26, 2011

13. International Consortium for Systemic Lupus Erythematosus Genetics (SLEGEN), Harley JB, Alarcón-Riquelme ME, Criswell LA, Jacob CO, Kimberly RP, Moser KL, Tsao BP, Vyse TJ, Langefeld CD, et al: Genome-wide association scan in women with systemic lupus erythematosus identifies susceptibility variants in ITGAM, PXK, KIAA1542 and other loci. Nat Genet 40: 204-210, 2008.

14. Graham RR, Cotsapas C, Davies L, Hackett R, Lessard CJ, Leon JM, Burtt NP, Guiducci C, Parkin M, Gates C, et al: Genetic variants near TNFAIP3 on $6 \mathrm{q} 23$ are associated with systemic lupus erythematosus. Nat Genet 40: 1059-1061, 2008.

15. Chai HC, Phipps ME, Othman I, Tan LP and Chua KH: HLA variants rs9271366 and rs9275328 are associated with systemic lupus erythematosus susceptibility in Malays and Chinese. Lupus 22: 198-204, 2013.
16. Morris DL, Taylor KE, Fernando MM, Nititham J, AlarcónRiquelme ME, Barcellos LF, Behrens TW, Cotsapas C, Gaffney PM, Graham RR, et al; International MHC and Autoimmunity Genetics Network; Systemic Lupus Erythematosus Genetics Consortium: Unraveling multiple MHC gene associations with systemic lupus erythematosus: model choice indicates a role for HLA alleles and non-HLA genes in Europeans. Am J Hum Genet 91: 778-793, 2012.

17. van Manen D, Kootstra NA, Boeser-Nunnink B, Handulle MA, van't Wout $\mathrm{AB}$ and Schuitemaker H: Association of HLA-C and HCP5 gene regions with the clinical course of HIV-1 infection. AIDS 23: 19-28, 2009.

18. Trachtenberg E, Bhattacharya T, Ladner M, Phair J, Erlich H and Wolinsky S: The HLA-B/-C haplotype block contains major determinants for host control of HIV. Genes Immun 10: 673-677, 2009.

19. Kurata R, Nakaoka H, Tajima A, Hosomichi K, Shiina T, Meguro A, Mizuki N, Ohono S, Inoue I and Inoko H: TRIM39 and RNF39 are associated with Behçet's disease independently of HLA-B-51 and -A-26. Biochem Biophys Res Commun 401: 533-537, 2010.

20. Aissani B, Boehme AK, Wiener HW, Shrestha S, Jacobson LP and Kaslow RA: SNP screening of central MHC-identified HLA-DMB as a candidate susceptibility gene for HIV-related Kaposi's sarcoma. Genes Immun 15: 424-429, 2014.

21. Glocker E, Ehl S and Grimbacher B: Common variable immunodeficiency in children. Curr Opin Pediatr 19: 685-692, 2007.

22. Hedrich CM, Crispin JC and Tsokos GC: Epigenetic regulation of cytokine expression in systemic lupus erythematosus with special focus on T cells. Autoimmunity 47: 234-241, 2014.

23. Lal G, Zhang N, van der Touw W, Ding Y, Ju W, Bottinger EP, Reid SP, Levy DE and Bromberg JS: Epigenetic regulation of Foxp3 expression in regulatory $\mathrm{T}$ cells by DNA methylation. $\mathrm{J}$ Immunol 182: 259-273, 2009.

24. Sullivan KE, Suriano A, Dietzmann K, Lin J, Goldman D and Petri MA: The TNF alpha locus is altered in monocytes from patients with systemic lupus erythematosus. Clin Immunol 123: 74-81, 2007.

25. Absher DM, Li X, Waite LL, Gibson A, Roberts K, Edberg J, Chatham WW and Kimberly RP: Genome-wide DNA methylation analysis of systemic lupus erythematosus reveals persistent hypomethylation of interferon genes and compositional changes to CD4 ${ }^{+}$T-cell populations. PLoS Genet 9: e1003678, 2013.

26. Lindberg J, af Klint E, Catrina AI, Nilsson P, Klareskog L, Ulfgren AK and Lundeberg J: Effect of infliximab on mRNA expression profiles in synovial tissue of rheumatoid arthritis patients. Arthritis Res Ther 8: R179, 2006.

27. Diehn M, Alizadeh AA, Rando OJ, Liu CL, Stankunas K, Botstein D, Crabtree GR and Brown PO: Genomic expression programs and the integration of the CD28 costimulatory signal in T cell activation. Proc Natl Acad Sci USA 99: 11796-11801, 2002.

28. Yan B, Tao ZF, Li XM, Zhang H, Yao J and Jiang Q: Aberrant expression of long noncoding RNAs in early diabetic retinopathy. Invest Ophthalmol Vis Sci 55: 941-951, 2014.

29. Haemmerle $M$ and Gutschner T: Long non-coding RNAs in cancer and development: where do we go from here? Int J Mol Sci 16: 1395-1405, 2015.

30. Scaruffi P, Stigliani S, Coco S, Valdora F, De Vecchi C, Bonassi S and Tonini GP: Transcribed-ultra conserved region expression profiling from low-input total RNA. BMC Genomics 11: 149, 2010.

31. Peng JC, Shen J and Ran ZH: Transcribed ultraconserved region in human cancers. RNA Biol 10: 1771-1777, 2013.

32. Sana J, Hankeova S, Svoboda M, Kiss I, Vyzula R and Slaby O: Expression levels of transcribed ultraconserved regions uc.73 and uc.388 are altered in colorectal cancer. Oncology 82: 114-118, 2012.

33. Lujambio A, Portela A, Liz J, Melo SA, Rossi S, Spizzo R, Croce CM, Calin GA and Esteller M: CpG island hypermethylation-associated silencing of non-coding RNAs transcribed from ultraconserved regions in human cancer. Oncogene 29: 6390-6401, 2010. 